\title{
How to Significantly Improve the Spectral Efficiency of Linear Modulations through Time-Frequency Packing and Advanced Processing
}

\author{
Andrea Modenini, ${ }^{1}$ Giulio Colavolpe,${ }^{1}$ and Nader Alagha ${ }^{2}$ \\ ${ }^{1}$ Università degli Studi di Parma (www.unipr.it), Dipartimento di Ingegneria dell'Informazione, Parma, Italy \\ ${ }^{2}$ ESTEC European Space Agency, Noordwijk, The Netherlands
}

\begin{abstract}
We recently investigated the spectral efficiency, achievable with a symbol-by-symbol receiver, for linear modulations employing time-frequency packing. In this paper, we will investigate the improvements that can be obtained by increasing the receiver complexity. In the numerical results, we will mainly concentrate on time packing (an extension of the so called fasterthan-Nyquist signaling technique) and on (i) receivers based on linear processing plus symbol-by-symbol detection or (ii) receivers based on a more sophisticated trellis processing with constrained complexity. Finally, the potential advantage of the proposed signalling scheme when combined with conventional low-density parity-check (LDPC) coding of the DVB-S2 air interface will be presented.
\end{abstract}

\section{INTRODUCTION}

In satellite links for broadcasting and broadband applications, orthogonal signaling, that ensures absence of intersymbol interference (ISI), is often adopted. As an example, in the 2nd-generation satellite digital video broadcasting (DVBS2) standard [1], a conventional square-root raised-cosine (RRC) pulse shaping filter is specified at the transmitter. In an additive white Gaussian noise channel and in the absence of other impairments, the use of a matched filter (MF) at the receiver and proper sampling ensure that optimal detection can be performed on a symbol-by-symbol basis. On the other hand, it is known that, when finite-order constellations are considered [e.g., phase-shift keying (PSK)], the efficiency of the communication system can be improved by giving up the orthogonality condition, thus accepting interference. For example, faster-than-Nyquist signaling (FTN, see [2], [3]) is a well known technique consisting of reducing the spacing between two adjacent pulses in the time-domain well below the Nyquist rate, thus introducing ISI. If the receiver is able to cope with the interference, the efficiency of the communication system will be increased. In the original papers on FTN signaling [2], [3], this optimal time spacing is obtained as the smallest value giving no reduction of the minimum Euclidean distance with respect to the Nyquist case. This ensures that, asymptotically, the ISI-free performance is reached, at least when the optimal detector is adopted. The i.u.d. capacity or information rate, i.e., the average mutual information when the channel inputs are independent and uniformly distributed (i.u.d.) random variables, is then computed, still assuming the adoption of the optimal detector [4], [5]. However, the complexity of this optimal detector easily becomes unmanageable, and no hints are provided on how to perform the optimization in the more practical scenario where a reduced-complexity receiver is employed.

In [6], a different approach for improving the spectral efficiency, that relies on both time packing of adjacent symbols and reducing the spacing of the adjacent channels when applicable (multi-carrier transmission), has been considered. It is assumed that, at the receiver side, a symbol-by-symbol detector working on the samples at the MF output is adopted, and the corresponding information rate is computed, by also optimizing time and frequency spacings to maximize the achievable spectral efficiency (ASE). Hence, rather than the minimum distance, the ASE is the performance measure and, in addition, a low-complexity detection algorithm, characterized by a given allowable complexity irrespectively of the interference set size, is considered at the receiver rather than the optimal detector employed in [2]-[5]. Although the MF output represents a set of sufficient statistics for optimal detection, a suboptimal symbol-by-symbol receiver is considered in [6]. Hence, the ASE can be improved by employing more sophisticated detection algorithms. In this paper, we will consider two cases: (i) a proper filtering of the MF output plus a symbol-by-symbol detector and (ii) the maximum $a$ posteriori (MAP) symbol detector that, in order to limit the receiver complexity, takes into account only a limited amount of interference.

This technique arises as a good alternative, for low-order constellations, to the shaping of the transmitted symbol distribution [7], providing spectral efficiencies that cannot be reached when orthogonal signaling is employed. Improving the ASE without increasing the constellation order can be considerably convenient since the larger the constellation size, the higher the decoding complexity and the lower the robustness to channel impairments such as time-varying phase noise and non-linearities. In the case of frequency packing, a further improvement could be achieved by adopting, at the receiver side, a multi-user detector, although this case is not considered in the numerical results for lack of space. The remainder of this paper is organized as follows. The system model is described in Section II. In Section III, we compute and optimize the spectral efficiency considering detectors with different complexity. Numerical results are reported in Section IV, where we also show the performance of some efficient modulation and coding formats (MODCODs) designed accordingly. Finally, some conclusions are drawn in Section V. 


\section{SySTEM MODEL}

We consider an additive white Gaussian noise (AWGN) channel and a frequency-division multiplexed system where perfectly synchronized (downlink assumption) adjacent channels employ the same linear modulation format, shaping pulse $p(t)$, and symbol interval (or time spacing) $T$. The shaping pulse is assumed to have unit energy. The received signal can be expressed as

$$
r(t)=\sqrt{2 E_{s}} \sum_{n} \sum_{\ell} x_{n, \ell} p(t-n T) e^{j 2 \pi \ell F t}+w(t)
$$

where $E_{s}$ is the symbol energy, $x_{n, \ell}$ the symbol transmitted over the $\ell$-th channel during the $n$-th symbol interval, $F$ the frequency spacing between adjacent channels, and $w(t)$ a circularly symmetric zero-mean white Gaussian noise process with power spectral density $2 N_{0}$. The transmitted symbols $\left\{x_{n, \ell}\right\}$ are independent and uniformly distributed and belong to a given zero-mean $M$-ary complex constellation $\chi$ properly normalized such that $E\left\{\left|x_{n, \ell}\right|^{2}\right\}=1$. Note that the summations in (1) extend from $-\infty$ to $+\infty$, namely an infinite number of time epochs and carriers are employed. For the spectral efficiency computation, we will consider the central user only using $F$ as a measure of the signal bandwidth.

The base pulse $p(t)$ has often RRC-shaped spectrum (RRC pulse in the following) with roll-off factor $\alpha$. In addition to it, we will consider other transmit pulses, e.g., a pulse whose spectrum is raised-cosine ( $\mathrm{RC}$ ) shaped ( $\mathrm{RC}$ pulse in the following) and a Gaussian pulse. In general, we will consider the case of time-frequency packing and we will optimize the frequency separation $F$ between two adjacent users and the symbol interval $T$ in order to maximize the ASE. In the case of bandlimited pulses (i.e., RRC and RC pulses), we will also consider time packing only. In this case, adjacent users are not allowed to overlap in frequency (i.e., $F=(1+\alpha) / T$ for $\mathrm{RRC}$ and RC pulses) and we may assume that only the user with $\ell=0$ is transmitted. In satellite communications, this can correspond to the use of a single carrier occupying the entire transponder bandwidth. This is of particular interest since the on-board power amplifier can operate closer to saturation and hence improve the efficiency.

\section{SPECTRAL EFFICIENCY OPTIMIZATION}

In this section, we shown how to compute the ASE for a given receiver and how to optimize the values of $T$ and $F$.

\section{A. Symbol-by-Symbol detection}

Let us consider the central user (i.e., that for $\ell=0$ ). We first consider the case shown in Figure 1(a) of a receiver composed by a filter matched to the shaping pulse $p(t)$, followed by a proper discrete-time filter, that works on $\eta \geq 1$ samples per symbol interval, and a symbol-by-symbol (SBS) detector. Although the discrete-time filter could be, in general, fractionally-spaced (FS, i.e., $\eta>1$ ), the detector will operate on one sample per symbol interval. These samples will be

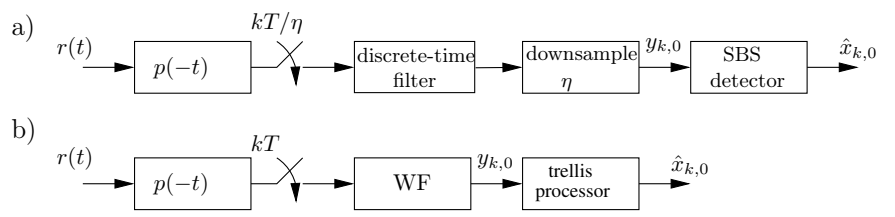

Figure 1. Some considered receivers: (a) symbol-by-symbol detector and (b) single-user detector based on trellis processing.

denoted by $\left\{y_{k, 0}\right\}$ and can be expressed as

$$
\begin{aligned}
y_{k, 0}= & \sqrt{2 E_{s}} x_{k, 0} h(0,0, k) \\
& +\sqrt{2 E_{s}} \sum_{(n, \ell) \neq(0,0)} x_{k-n, \ell} h(n, \ell, k)+z_{k}
\end{aligned}
$$

in which $h(n, \ell, k)$ is the residual interference at time $k T$ due to the $\ell$-th user and the $(k-n)$-th transmitted symbol, and $\left\{z_{k}\right\}$ is the additive noise term, in general colored unless a whitening filter (WF) is employed after the MF. The discretetime filter is assumed properly normalized such that the noise variance is $2 N_{0}$. The dependence of coefficients $h(n, \ell, k)$ on $k$ is through a complex coefficient of unit amplitude which disappears for $\ell=0$ (hence $h(n, 0, k)$ is independent of $k$ ) and is due to the fact that $F$ is not an integer multiple of $1 / T$.

Eq. (2) shows the two different impairments experienced by the receiver, namely the background noise and the interference. Instead of simply neglecting the interference due to adjacent symbols and users, we pursue here a more general approach, which consists of modeling the interference as a zero-mean Gaussian process with power spectral density equal to $2 N_{I}$, of course independent of the additive thermal noisewe point out that this approximation is exploited only by the receiver, while in the actual channel the interference is clearly generated as in (2). Note that the interference is really Gaussian distributed only if the transmitted symbols $x_{k, \ell}$ are Gaussian distributed as well. However, especially when the interference set is small, e.g., when $T$ and $F$ are large, the actual interference distribution may substantially differ from a Gaussian distribution.

We define auxiliary channel the channel model assumed by the receiver. With the above mentioned Gaussian approximation, the auxiliary channel is

$$
y_{k, 0}=\sqrt{2 E_{s}} x_{k, 0} h(0,0, k)+v_{k}
$$

where $\left\{v_{k}\right\}$ are independent and identically distributed zeromean circularly symmetric Gaussian random variables, with variance $2\left(N_{0}+N_{I}\right)$. It turns out that

$$
N_{I}=E_{s} \sum_{(n, \ell) \neq(0,0)}|h(n, \ell, k)|^{2}
$$

which results to be independent of $k$, as can be easily shown. We are interested in evaluating the ultimate performance limits achievable by a symbol-by-symbol receiver designed for the auxiliary channel (3) when the actual channel is that in (2), in terms of information rate (or spectral efficiency). This issue is an instance of mismatched detection [8] (see also [9]). The achievable information rate (AIR), measured in bit per channel 
use, for this mismatched receiver is

$$
I\left(x_{k, 0} ; y_{k, 0}\right)=E_{x_{k, 0}, y_{k, 0}}\left\{\log _{2} \frac{M p_{Y_{k, 0} \mid X_{k, 0}}\left(y_{k, 0} \mid x_{k, 0}\right)}{\sum_{x \in \chi} p_{Y_{k, 0} \mid \chi}\left(y_{k, 0} \mid x\right)}\right\}
$$

where $p_{Y_{k, 0} \mid X_{k, 0}}\left(y_{k, 0} \mid x_{k, 0}\right)$ is a Gaussian probability density function (PDF) of mean $x_{k, 0}$ and variance $2\left(N_{0}+N_{I}\right)$ [in accordance with the auxiliary channel model (3)], while the outer statistical average, with respect to $x_{k, 0}$ and $y_{k, 0}$, is carried out according to the real channel model (2) [9]. Eq. (5) can be evaluated efficiently by means of a Monte Carlo average [9]. From a system viewpoint, the spectral efficiency, that is the amount of information transmitted per second and per Hertz, is a more significant quality figure than the information rate. Under the assumption of infinite transmission, the ASE is defined as

$$
\eta=\frac{1}{F T} I\left(x_{k, 0} ; y_{k, 0}\right) \quad\left[\frac{\mathrm{bit}}{\mathrm{s} \cdot \mathrm{Hz}}\right] \text {. }
$$

For a given constellation and shaping pulse, it is possible to find the spacings $T$ and $F$ that provide the largest ASE. In general, we could expect that the optimal spacings depend on the signal-to-noise ratio (SNR). In fact, it is possible to show that, as the SNR increases, not only does the ASE increase, but also the optimal values of the spacings change. The properties of the function $\eta\left(T, F, E_{S} / N_{0}\right)$ cannot be easily studied in closed form, but it is clear, by physical arguments, that it is bounded, continuous in $T$ and $F$, and tends to zero when $T, F \rightarrow 0$ or $T, F \rightarrow \infty$. Hence, the function $\eta(T, F)$ has a maximum value-according to our findings, in most cases there are no local maxima other than the global maximum. Formally, for a given modulation format, shaping pulse, and value of $E_{S} / N_{0}$, the optimization problem consists of finding the maximum of $\eta\left(T, F, E_{S} / N_{0}\right)$ varying $T$ and $F$. This problem can be solved by evaluating $\eta\left(T, F, E_{S} / N_{0}\right)$ on a grid of values of $T$ and $F$ (coarse search), followed by an interpolation of the obtained values (fine search).

A measure of the SNR more significant than $E_{s} / N_{0}$ is given by $E_{b} / N_{0}$, being $E_{b}$ the mean energy per information bit, for which $E_{s}=I\left(E_{s}\right) E_{b}$ holds. The optimization problem becomes

$$
\eta_{\mathbf{M}}\left(E_{b} / N_{0}\right)=\max _{T, F>0} \eta\left(T, F, E_{b} / N_{0}\right)
$$

In order to solve it for a given value of $E_{b} / N_{0}$, we employed the following technique. The AIR is first evaluated for some values of the couple $(T, F)$, and $E_{s} / N_{0}$. The two sets, including their cardinalities, must be designed so as to ensure an accurate sampling of the AIR, when the latter is interpreted as a function of $T, F$, and $E_{s} / N_{0}$. For each couple $\left(T_{i}, F_{j}\right)$, cubic spline interpolation can be used to obtain a continuous function of $E_{s} / N_{0}$ (fine search), denoted as $I\left(T_{i}, F_{j}, E_{s} / N_{0}\right)$. Then, given a value of $E_{b} / N_{0}$ the following fixed-point problems are solved in $E_{s} / N_{0}$ for different couples $\left(T_{i}, F_{j}\right)$,

$$
\frac{E_{s}}{N_{0}}=I\left(T_{i}, F_{j}, \frac{E_{s}}{N_{0}}\right) \frac{E_{b}}{N_{0}}
$$

and the AIRs corresponding to the solutions are denoted by $I\left(T_{i}, F_{j}, E_{b} / N_{0}\right)$. Further improvements could be achieved by adding $N_{I}$ as variable in eq. (7). However, we have found by numerical results that choosing $N_{I}$ as in (4) is almost optimal.

The spectral efficiency depends on the employed discretetime filter. Since the optimization of this filter with the aim of maximizing the spectral efficiency is a hard task, we restricted our analysis to the cases of a WF, that will be also considered in Section III-B, and of a minimum mean square error (MMSE) feedforward equalizer, possibly fractionally spaced (FS) with at most 22 taps.

\section{B. Single-User Trellis Processing}

Improved, still achievable, lower bounds can be obtained by relaxing the constraint on the adopted detection algorithm. In other words, we can consider a more complex receiver able to cope with (a portion of) the interference introduced by the adoption of the time-frequency packing. The receiver considered in this section will not cope with the interference due to the adjacent users - a single-user receiver is still adopted.

For a general channel with finite intersymbol interference, an optimal MAP symbol detector can be designed working on the samples at the WF output as shown in Figure 1(b). These samples, denoted to as Forney observation model [10], can still be expressed as in (2) with a proper expression of coefficients $h(n, \ell, k)$. We assume to adopt the optimal receiver for the following auxiliary channel:

$$
y_{k, 0}=\sqrt{2 E_{s}} \sum_{0 \leq n \leq L} f_{n} x_{k-n, 0}+v_{k}
$$

where $\left\{f_{n}\right\}_{n \geq 0}$ are such that $f_{n}=h(n, 0, k)$ and, as mentioned, are independent of $k$, whereas the noise samples $\left\{v_{n}\right\}$, that take into account the white noise and the residual interference, are assumed independent and identically distributed zero-mean circularly symmetric Gaussian random variables with variance $2\left(N_{0}+N_{I}\right)$, with

$$
N_{I}=\sum_{n>L} E_{s}\left|f_{n}\right|^{2}+\sum_{n} \sum_{\ell \neq 0} E_{s}|h(n, \ell, k)|^{2} .
$$

which is still independent of $k$. The corresponding MAP symbol detector takes the form of the classical algorithm by Bahl, Cocke, Jelinek and Raviv (BCJR) [11] working on a trellis whose state takes into account $L$ interfering symbols only, according to a given maximal allowable receiver complexity. The number of trellis states is equal to $S=M^{L}$.

Let us define $\mathbf{x}^{N}=\left(x_{0,0}, x_{1,0}, \ldots, x_{N, 0}\right)$ and $\mathbf{y}^{N}=$ $\left(y_{0,0}, y_{1,0}, \ldots, y_{N, 0}\right), N$ being a proper integer. The simulationbased method described in [9] allows to evaluate the AIR for the mismatched receiver, i.e.,

$$
\begin{aligned}
I(\mathbf{x} ; \mathbf{y}) & =\lim _{N \rightarrow+\infty} \frac{1}{N} I\left(\mathbf{x}^{N} ; \mathbf{y}^{N}\right) \\
& =\lim _{N \rightarrow+\infty} \frac{1}{N} E\left\{\log _{2} \frac{p\left(\mathbf{y}^{N} \mid \mathbf{x}^{N}\right)}{p\left(\mathbf{y}^{N}\right)}\right\}\left[\frac{\text { bit }}{\text { ch. use }}\right](10)
\end{aligned}
$$




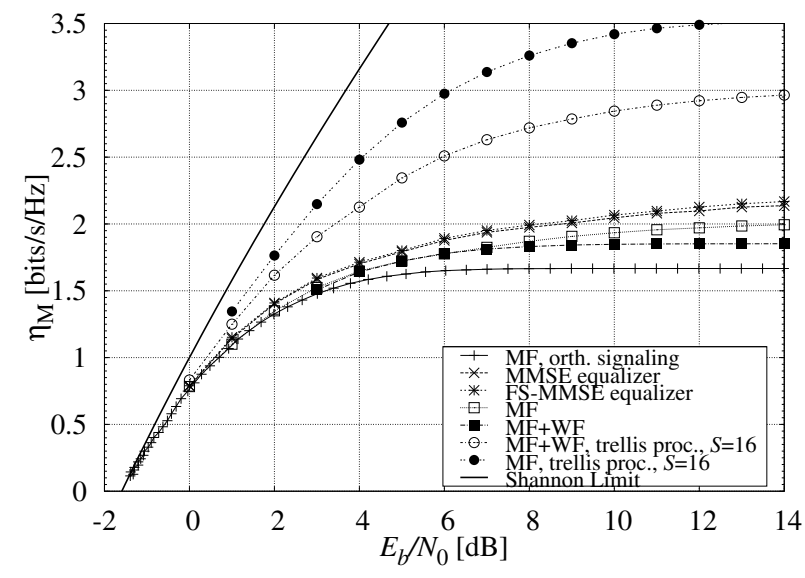

Figure 2. ASE for QPSK with Gray mapping and a RRC pulse having $\alpha=0.2$.

In (10), $p\left(\mathbf{y}^{N} \mid \mathbf{x}^{N}\right)$ and $p\left(\mathbf{y}^{N}\right)$ are PDF according to the auxiliary channel model, while the outer statistical average is with respect to the input and output sequences evaluated according to the actual channel model [9]. Eq. (10) can be evaluated recursively through the forward recursion of the BCJR detection algorithm matched to the auxiliary channel model [9]. Once the AIR has been computed, the spectral efficiency can be derived and the optimal time and frequency spacings optimized accordingly, as described in the previous section. For channels with finite ISI, optimal MAP symbol detection can be equivalently implemented by working directly on the MF output [12], i.e., on the so-called Ungerboeck observation model [13]. The equivalence does not hold when reduced-complexity detection is considered and interference from adjacent channels arises. Since it is difficult to predict which is the most convenient observation model, it is of interest to evaluate the ASE when both models are employed and this can be done as described for the Forney model (see also [14] for details).

\section{Multi-User Detection}

Although the assumption of a single-user auxiliary channel gives very useful results, tighter lower bounds can be obtained by using a more general auxiliary channel model. In fact, we can consider a receiver for the central user (that with $\ell=$ 0 ) that, in addition to the interference taken into account by the receivers in Section III-B, also takes into account the $J$ adjacent signals on each side as well (multi-user receiver)—we again point out that this approximation is exploited only by the receiver, while in the actual channel the interference is generated as in (1). The exact MAP receiver for the multiuser auxiliary channel can be easily derived and employed to find the ASE in the new scenario. The benefit of employing the multi-user auxiliary channel model when evaluating the ASE is two-fold: first, it allows to evaluate the performance degradation due to the use of single-user receivers, despite the presence of a strong adjacent channel interference, with respect to a more involved multi-user receiver, which is more "matched" to the real channel. Second, it gives a practical performance upper bound when low-complexity approximate multi-user receivers, for example based on linear equalization

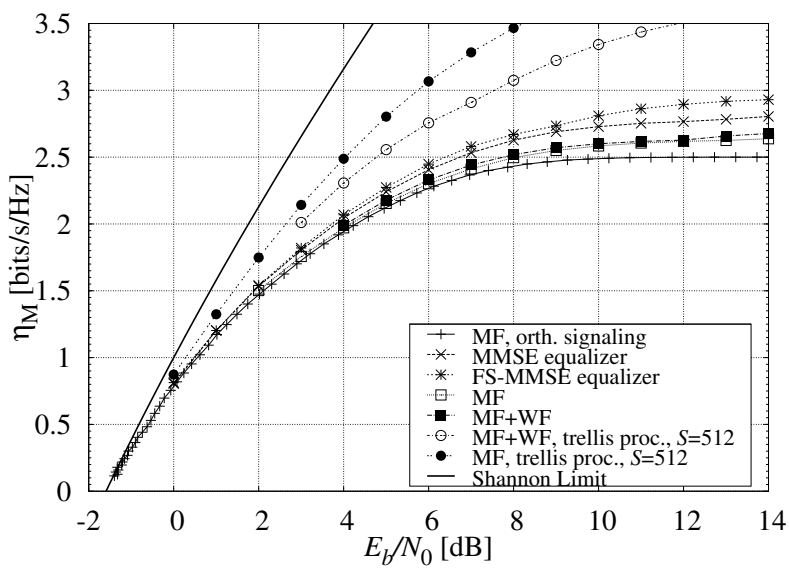

Figure 3. ASE for 8-PSK with a RRC pulse having $\alpha=0.2$.

or interference cancellation, are employed (as examples, those in [15] and references therein). Obviously, in this case some (limited) degradation must be expected. For lack of space, in this paper we will not consider further this option.

\section{Simulation Results}

In this section, we report the optimized spectral efficiency $\eta_{\mathrm{M}}$ as a function of $E_{b} / N_{0}$ for different modulation formats and shaping pulses. The considered modulation formats are the quaternary and octal PSK (QPSK and 8-PSK).

Fig. 2 shows the optimized ASE in case of time packing only for the QPSK modulation with a RRC pulse of rolloff $\alpha=0.2$. Both symbol-by-symbol detection and trellis processing (this latter taking into account $L=4$ interfering symbols) are considered assuming Gray mapping. In this case, at the receiver side we may use two identical and independent detectors, one working on the in-phase and the other one on the quadrature component. This is beneficial in case of adoption of a MAP symbol detector. In fact, when $L$ interfering symbols are taken into account, we have two detectors working on a trellis with $2^{L}$ states instead of a single detector working on a trellis with $4^{L}$ states. Hence, for a given complexity, a larger number of interferers can be taken into account. The curve related to the absence of time packing (i.e., in case of orthogonal signaling) and the Shannon Limit for AWGN [16], are also shown for comparison. It can be observed that the time-packing technique allows to improve the spectral efficiency for each $E_{b} / N_{0}$ value with respect to the case of orthogonal signaling. Moreover it can noticed that, in case of use of a symbol-by-symbol detector, the FS-MMSE equalizer seems the best option whereas the Ungerboeck observation model is more suited in case of trellis processing. Similar considerations hold for the 8-PSK modulation with a RRC pulse of $\alpha=0.2$. The relevant results are shown in Fig. 3. Still considering QPSK with Gray mapping and trellis processing with $S=16$, we evaluated the effect of different shaping pulses. In particular, RRC and RC pulses with different rolloff factors have been considered along with prolate spheroidal wave functions [17] and the Gaussian pulse. In these two latter cases, frequency packing is also employed. Fig. 4 shows the performance of some of the considered pulses. In particular, 


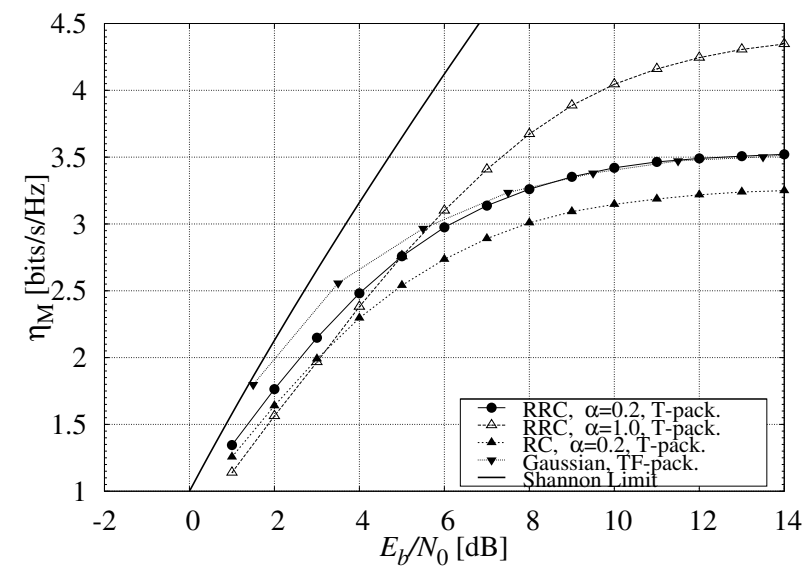

Figure 4. ASE for QPSK with Gray mapping by using different pulses. At the receiver, a MF front end and trellis processing with $S=16$ is considered.

RRC pulses with $\alpha$ equal to 0.2 and 1.0 outperform all other pulses at low and high $E_{b} / N_{0}$ values, respectively. In particular, an impressive asymptotic spectral efficiency of 4.3 $\mathrm{bit} / \mathrm{s} / \mathrm{Hz}$ is obtained with QPSK and $\alpha=1 .{ }^{1}$ Hence, it seems that there is no advantage in replacing the RRC with other pulses, at least when a single-user receiver is considered. Some improvements could, in fact, be obtained by adopting multiuser detection at the receiver.

What information theory promises can be approached by using proper coding schemes. We considered MODCODs using the low-density parity-check (LDPC) codes with length 64,800 bits of the DVB-S2 standard [1], properly combined with QPSK and 8-PSK modulations with time packing. RRC pulses with $\alpha=0.2$ or $\alpha=1$ are considered. The corresponding packet error rate (PER) have been computed by means of Monte Carlo simulations and the results are reported in the spectral efficiency plane in Fig. 5 using, as reference, an MPEG PER of $10^{-4}$. In the same figure, the performance of the MODCODs based on the same LDPC codes with orthogonal signaling and employing QPSK, 8-PSK, and the amplitude phase-shift keying (APSK) modulation with 16 and 32 symbols (16- and 32-APSK) [1], are also shown for comparison. We can observe that we can reach, with QPSK, values of spectral efficiency that, in case of orthogonal signaling, cannot be reached even with 16-APSK.

\section{Conclusions}

We have investigated a way to improve the spectral efficiency of low-order linear modulations with different receivers. The improvement is related to the use of time-frequency packing and the adoption of detectors with different complexity. We accordingly designed proper MODCODs, showing that large benefits can be obtained even by simply using time packing.

\section{ACKNOWLEDGEMENT}

This work is funded by the European Space Agency, ESA-ESTEC, Noordwijk, The Netherlands, under contract no. 4000102300.

\footnotetext{
${ }^{1}$ This is due to the fact that the shaping pulse is smoother and so, for a given value of $T$, the introduced interference is lower.
}

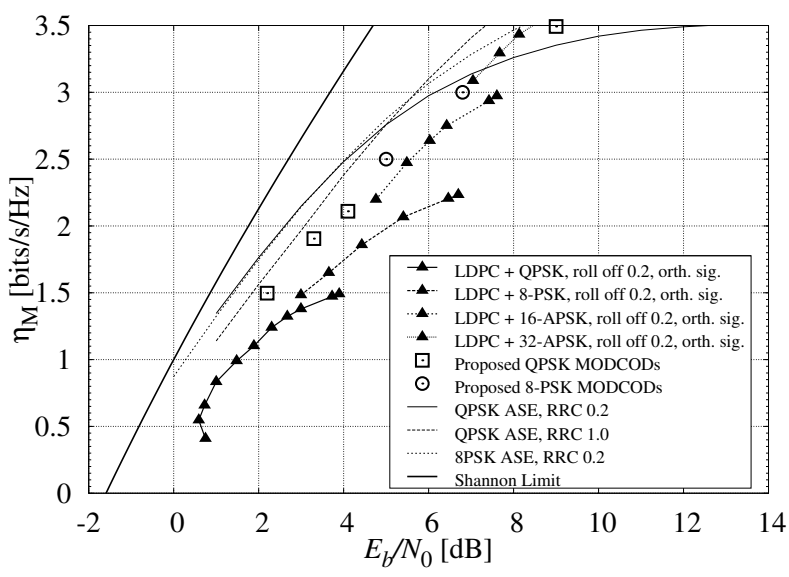

Figure 5. Designed MODCODs for QPSK and 8PSK with RRC pulses.

\section{REFERENCES}

[1] ETSI EN 301307 Digital Video Broadcasting (DVB); V1.1.2 (2006-06), Second generation framing structure, channel coding and modulation systems for Broadcasting, Interactive Services, News Gathering and other Broadband satellite applications, 2006.

[2] J. E. Mazo, "Faster-than-Nyquist signaling," Bell System Tech. J., vol. 54, pp. 1450-1462, Oct. 1975.

[3] J. E. Mazo and H. J. Landau, "On the minimum distance problem for faster-than-Nyquist signaling," IEEE Trans. Inform. Theory, pp. 14201427, Nov. 1988

[4] F. Rusek and J. B. Anderson, "On information rates of faster than Nyquist signaling," in Proc. IEEE Global Telecommun. Conf., (San Francisco, CA, U.S.A.), Nov. 2006.

[5] F. Rusek and J. B. Anderson, "Maximal capacity partial response signaling," in Proc. IEEE Intern. Conf. Commun., pp. 821-826, June 2007.

[6] A. Barbieri, D. Fertonani, and G. Colavolpe, "Time-frequency packing for linear modulations: spectral efficiency and practical detection schemes," IEEE Trans. Commun., vol. 57, pp. 2951-2959, Oct. 2009.

[7] A. R. Calderbank and L. H. Ozarow, "Nonequiprobable signalling on the Gaussian channel," IEEE Trans. Inform. Theory, vol. 36, pp. 726-740, July 1990.

[8] N. Merhav, G. Kaplan, A. Lapidoth, and S. Shamai, "On information rates for mismatched decoders," IEEE Trans. Inform. Theory, vol. 40, pp. 1953-1967, Nov. 1994.

[9] D. M. Arnold, H.-A. Loeliger, P. O. Vontobel, A. Kavčić, and W. Zeng, "Simulation-based computation of information rates for channels with memory," IEEE Trans. Inform. Theory, vol. 52, pp. 3498-3508, Aug. 2006.

[10] G. D. Forney, Jr., "Maximum-likelihood sequence estimation of digital sequences in the presence of intersymbol interference," IEEE Trans. Inform. Theory, vol. 18, pp. 284-287, May 1972.

[11] L. R. Bahl, J. Cocke, F. Jelinek, and J. Raviv, "Optimal decoding of linear codes for minimizing symbol error rate," IEEE Trans. Inform. Theory, vol. 20, pp. 284-287, Mar. 1974.

[12] G. Colavolpe and A. Barbieri, "On MAP symbol detection for ISI channels using the Ungerboeck observation model," IEEE Commun. Letters, vol. 9, pp. 720-722, Aug. 2005.

[13] G. Ungerboeck, "Adaptive maximum likelihood receiver for carriermodulated data-transmission systems," IEEE Trans. Commun., vol. com22, pp. 624-636, May 1974.

[14] F. Rusek and D. Fertonani, "Lower bounds on the information rate of intersymbol interference channels based on the ungerboeck observation model," in Proc. IEEE International Symposium on Information Theory, 2009.

[15] G. Colavolpe, D. Fertonani, and A. Piemontese, "SISO detection over linear channels with linear complexity in the number of interferers," IEEE J. of Sel. Topics in Signal Proc., vol. 5, pp. 1475-1485, Dec. 2011.

[16] C. Shannon, "A mathematical theory of communication," Bell System Tech. J., pp. 379-423, July 1948.

[17] D. Slepian and H. O. Pollak, "Prolate spheroidal wave functions, Fourier analysis and uncertainty - I," Bell System Tech. J., vol. 40, pp. 43-63, Jan. 1961 